\title{
Heisenberg Model with Added Dzyaloshinskii-Moria Interaction
}

\author{
M. R. Soltani, ${ }^{1}$ J. Vahedi, ${ }^{1}$ M. R. Abolhassani, ${ }^{1}$ and A. A. Masoudi ${ }^{2}$ \\ ${ }^{1}$ Department of Physics, Science and Research Branch, Islamic Azad University, Tehran, Iran \\ ${ }^{2}$ Department of Physics, Alzahra University, P.O. Box 19585-466, Tehran, Iran
}

Correspondence should be addressed to M. R. Soltani, m.r.soltani.em@gmail.com

Received 25 October 2011; Accepted 30 November 2011

Academic Editors: C. Andreani and W. Selke

Copyright (C) 2011 M. R. Soltani et al. This is an open access article distributed under the Creative Commons Attribution License, which permits unrestricted use, distribution, and reproduction in any medium, provided the original work is properly cited.

\begin{abstract}
We have considered the 1D spin-(1/2) Heisenberg model with added Dzyaloshinskii-Moriya interaction. The effect of a uniform magnetic field on the ground state phase diagram of the model is studied. We have mapped the model to an effective model which is known as the 1D XXZ in both uniform and staggered magnetic fields. By selecting a block of two or three spins, we have solved the Hamiltonian exactly. Our results show that the quantum phase transitions can be obtained from the block of pair or three spins.
\end{abstract}

\section{Introduction}

Study of the magnetic field effect on the ground state characteristics of chain model of antiferromagnetic (AF) spin-(1/2) has attracted much interest in recent years. The Hamiltonian of this model in a homogenous magnetic field $h$ is given by

$$
H=J \sum_{j=1}^{N}\left(S_{j}^{x} S_{j+1}^{x}+S_{j}^{y} S_{j+1}^{y}+S_{j}^{z} S_{j+1}^{z}\right)+h \sum_{j=1}^{N} S_{j}^{x},
$$

where $J>0$ is the exchange coupling and $h$ denotes the magnetic field. The exact solution for $h=0$ is obtained by Bethe ansatz [1]. The energy spectrum is gapless and the system is in the Luttinger liquid phase. In this phase, the spin correlation functions are in power form. When the system imposes by homogeneous magnetic field, its spectrum remains gapless up to the critical field $h_{c}=2 J$. Here the Pokrovsky-Talapov phase transition takes place and the ground state of the system becomes saturated ferromagnetic $[2,3]$. In many experimental data, the resulting data are different by theoretical predications [4-10]. These differences are due to DM interaction $[11,12]$, its Hamiltonian can be written as follows:

$$
\begin{aligned}
H= & J \sum_{j=1}^{N}\left(S_{j}^{x} S_{j+1}^{x}+S_{j}^{y} S_{j+1}^{y}+S_{j}^{z} S_{j+1}^{z}\right) \\
& +\sum_{j=1}^{N} \vec{D} \cdot\left(\vec{S}_{j} \times \vec{S}_{j+1}\right)+h \sum_{j=1}^{N} S_{j}^{x},
\end{aligned}
$$

where $\vec{D}$ is the DM vector and chooses in the $z$ direction. [As DM interaction broken the fundamental SU(2) symmetry which is related to Heisenberg isotropic interaction]. It is known as the origin of many declination and creates many different qualitative effects. It specially creates an energy gap by the scale of gap $\approx(D h)^{2 / 3}[13,14]$. DM interaction can act as a vector potential on the spin wave in the magnon spin Hall effect [15]. In ferromagnetic nanowires DM interaction has profound effect on the motion of domain walls [16]. It can also give rise to spin current and soliton in spin chains [17]. We emphasis that the studies of Heisenberg model commonly without DM interaction and the role of DM interaction on GS of Heisenberg model of AF by spin-( $1 / 2)$ are considered less than the other models. As the exact solution of this model with DM interaction cannot be done, we need to do a great value of theoretical works.

\section{Mapping to the XXZ Chain}

In this section we try to map the Heisenberg chain in the presence of external magnetic field and DM interaction to the well-known XXZ chain, analytically. At first we have done a $\pi / 2$ rotation around $\mathrm{X}$, to convert $z \rightarrow y, y \rightarrow z$ :

$$
\begin{aligned}
H= & J \sum_{j=1}^{N}\left(S_{j}^{x} S_{j+1}^{x}+S_{j}^{y} S_{j+1}^{y}+S_{j}^{z} S_{j+1}^{z}\right) \\
& +D \sum_{j=1}^{N}\left(S_{j}^{x} S_{j+1}^{y}-S_{j}^{y} S_{j+1}^{x}\right)+h \sum_{j=1}^{N} S_{j}^{x} .
\end{aligned}
$$


Using $S^{ \pm}$operators [15],

$S_{j}^{ \pm}=S_{j}^{x} \pm S_{j}^{y} \Longrightarrow S_{j}^{x}=\frac{1}{2}\left(S_{j}^{+}+S_{j}^{-}\right), \quad S_{j}^{y}=\frac{1}{2 i}\left(S_{j}^{+}-S_{j}^{-}\right)$. follows:

The interacting Hamiltonian terms can be calculated as

$$
\begin{aligned}
S_{j}^{x} S_{j+1}^{y} & =\frac{1}{4 i}\left(S_{j}^{+}+S_{j}^{-}\right)\left(S_{j+1}^{+}-S_{j+1}^{-}\right) \\
& =\frac{1}{4 i}\left(S_{j}^{+} S_{j+1}^{+}-S_{j}^{+} S_{j+1}^{-}+S_{j}^{-} S_{j+1}^{+}-S_{j}^{-} S_{j+1}^{-}\right), \\
S_{j}^{y} S_{j+1}^{x} & =\frac{1}{4 i}\left(S_{j}^{+}-S_{j}^{-}\right)\left(S_{j+1}^{+}+S_{j+1}^{-}\right) \\
& =\frac{1}{4 i}\left(S_{j}^{+} S_{j+1}^{+}+S_{j}^{+} S_{j+1}^{-}-S_{j}^{-} S_{j+1}^{+}-S_{j}^{-} S_{j+1}^{-}\right) \\
S_{j}^{x} S_{j+1}^{x} & =\frac{1}{4}\left(S_{j}^{+}+S_{j}^{-}\right)\left(S_{j+1}^{+}+S_{j+1}^{-}\right) \\
& =\frac{1}{4}\left(S_{j}^{+} S_{j+1}^{+}+S_{j}^{+} S_{j+1}^{-}+S_{j}^{-} S_{j+1}^{+}+S_{j}^{-} S_{j+1}^{-}\right) \\
S_{j}^{y} S_{j+1}^{y} & =\frac{1}{4 i}\left(S_{j}^{+}-S_{j}^{-}\right)\left(S_{j+1}^{+}-S_{j+1}^{-}\right) \\
& =\frac{1}{4}\left(-S_{j}^{+} S_{j+1}^{+}+S_{j}^{+} S_{j+1}^{-}+S_{j}^{-} S_{j+1}^{+}-S_{j}^{-} S_{j+1}^{-}\right) \\
S_{j}^{x} S_{j+1}^{x}+S_{j}^{y} S_{j+1}^{y} & =\frac{1}{2}\left(S_{j}^{+} S_{j+1}^{-}+S_{j}^{-} S_{j+1}^{+}\right) .
\end{aligned}
$$

Substituting these terms, we simply obtain the following:

$$
\begin{aligned}
H^{\mathrm{DM}} & =D \sum_{j=1}^{N}\left(S_{j}^{x} S_{j+1}^{y}-S_{j}^{y} S_{j+1}^{x}\right) \\
& =\frac{D}{2 i} \sum_{j=1}^{N}\left(S_{j}^{-} S_{j+1}^{+}-S_{j}^{+} S_{j+1}^{-}\right), \\
H^{\mathrm{XX} x} & =\frac{J}{2} \sum_{j=1}^{N}\left(S_{j}^{+} S_{j+1}^{-}+S_{j}^{-} S_{j+1}^{+}+2 S_{j}^{z} S_{j+1}^{z}\right) .
\end{aligned}
$$

Finally one can find the effective Hamiltonian:

$$
\begin{aligned}
H_{1}= & \frac{J}{\cos \varphi} \sum_{j=1}^{N}\left(\left(S_{j}^{x} S_{j+1}^{x}+S_{j}^{y} S_{j+1}^{y}\right)+\cos \varphi S_{j}^{z} S_{j+1}^{z}\right) \\
& -h \sum_{j=1}^{N} S_{j}^{x}-h^{\prime} \sum_{J=1}^{N}(-1)^{j} S_{j}^{y},
\end{aligned}
$$

in which $h^{\prime} \propto \vec{D} \times \vec{h}$ is known as staggered field and $D=J \tan \varphi$. This Hamiltonian is considered as XXZ chain Hamiltonian in homogenous and staggered field. It can be seen that AF can be created by DM interaction. It is shown theoretically that applying a staggered field on the AF chain of Heisenberg spin-(1/2), causes an energy gap in the spectrum of the system. In the absences of homogenous and staggered magnetic field $\left(h=h^{\prime}=0\right)$ the GS of the system is in the spin Luttinger liquid phase. In the presence of homogenous field, the exact solution is obtained by the Bethe ansatz methods. In the presence of staggered magnetic field, the exact solution is impossible. AF causes Neel ordered in $y$ direction (unrotated $\mathrm{z}$ direction). When $h^{\prime}=0, h \neq 0$, the magnetization in the $h$ direction is an uniform and increasing function of $h$.

In what follows, we will consider two- and three-particle systems because they can be exactly calculated, and this phenomena takes place in some alternative spin chain that gives us a qualitative view of real interacting model which cannot be solved.

\section{Two Particles Spin-(1/2) Systems}

In this section we consider a special model which composed of two particle systems. We choose AF interacting for spins.

$$
H=J\left(\overrightarrow{S_{1}} \cdot \overrightarrow{S_{2}}\right)+D\left(\overrightarrow{S_{1}} \times \overrightarrow{S_{2}}\right)-h\left(S_{1}^{x}+S_{2}^{x}\right) .
$$

To solve this, we choose the singlet and triplet states for Hilbert space. We know that its eigenstates relate to $S_{1}^{z} S_{2}^{z}$ as follows:

$$
\begin{array}{ll}
S_{1}^{z} S_{2}^{z}|\uparrow \uparrow\rangle=\frac{1}{4}|\uparrow \uparrow\rangle, & S_{1}^{z} S_{2}^{z}|\uparrow \downarrow\rangle=-\frac{1}{4}|\uparrow \downarrow\rangle, \\
S_{1}^{z} S_{2}^{z}|\downarrow \uparrow\rangle=-\frac{1}{4}|\downarrow \uparrow\rangle, & S_{1}^{z} S_{2}^{z}|\downarrow \downarrow\rangle=\frac{1}{4}|\downarrow \downarrow\rangle .
\end{array}
$$

Using these eigenstates, the singlet and triplet states are defined as follows:

$$
\begin{aligned}
|S\rangle & =\frac{1}{\sqrt{2}}(|\uparrow \downarrow\rangle-|\downarrow \uparrow\rangle), \\
\left|t_{1}\right\rangle & =|\uparrow \uparrow\rangle, \\
\left|t_{0}\right\rangle & =\frac{1}{\sqrt{2}}(|\uparrow \downarrow\rangle+|\downarrow \uparrow\rangle), \\
\left|t_{-1}\right\rangle & =|\downarrow \downarrow\rangle
\end{aligned}
$$

In this [base kets], the matrix representation of Hamiltonian is as follows:

$$
H=\left(\begin{array}{cccc}
-\frac{3}{4} J & -\frac{D}{2 \sqrt{2}} & 0 & -\frac{D}{2 \sqrt{2}} \\
-\frac{D}{2 \sqrt{2}} & \frac{J}{4} & -\frac{\sqrt{2}}{2} h & 0 \\
0 & -\frac{\sqrt{2}}{2} h & \frac{J}{4} & -\frac{\sqrt{2}}{2} h \\
-\frac{D}{2 \sqrt{2}} & 0 & -\frac{\sqrt{2}}{2} h & \frac{J}{4}
\end{array}\right) .
$$

We diagonalize the Hamiltonian (11) and determine the GS of the systems as follows:

$$
|G S\rangle=A|S\rangle+B\left(\left|t_{1}\right\rangle+\left|t_{-1}\right\rangle\right)+C\left|t_{0}\right\rangle .
$$

In which $A, B$, and $C$ are as follows:

$$
A=\frac{J}{4}, \quad B=\frac{D}{2 \sqrt{2}}, \quad C=\frac{\sqrt{2} h}{2} .
$$


In order to define the characteristics of the GS of the model in different subspaces of the GS phase diagram, we first calculated the magnetic order parameter $M^{x, y, z}$ and $M_{s t}^{x, y, z}$ as follows:

$$
\begin{gathered}
M^{x, y, z}=\left\langle\mathrm{GS}\left|\frac{1}{N} \sum_{n} S_{n}^{x, y, z}\right| \mathrm{GS}\right\rangle, \\
M_{s t}^{x, y, z}=\left\langle\mathrm{GS}\left|\frac{1}{N} \sum_{n}(-1)^{n} S_{n}^{x, y, z}\right| \mathrm{GS}\right\rangle .
\end{gathered}
$$

The averaging is calculated on GS. The magnetization along the applied field is plotted via $h$ for different DM vectors in Figure 1. It can be seen that for small $D=0.1$, the magnetization for small fields remains close to zero. Increasing $h$ up to $h>h_{c}$, the magnetization becomes saturated. Due to quantum fluctuations in the presence of $D$, there is not any sharp transient in saturation point. For greater values of $D$, the magnetization increases by imposing magnetic field. In XXZ model, in $h<h_{c}$ magnetization has some fluctuations and for $h>h_{c}$ will be saturated. Imposing transverse external magnetic field, causes removing quantum fluctuation and the spins completely direct along the field, but the magnetization saturation cannot be seen because of broken symmetry in finite systems.

We show that in previous section that by imposing magnetic field $h$ in the presence of DM interaction a staggered magnetization will be created normal to $\vec{D}-\vec{h}$ surface using the definition of staggered magnetization we can obtain the following:

$$
M_{s t}^{z}=\frac{1}{2}\left\langle G S\left|S_{1}^{z}-S_{2}^{z}\right| G S\right\rangle .
$$

The staggered magnetization via $h$ for $D=0.1,0.5,1.0$. is plotted in Figure 2. Figure 2 shows that applying homogeneous $h$, creates Neel order in $z$ direction. That shows spin-flop ordering. There is a maximum for AM about 0.3. This is the same maximum of an independent dimer which weakly depends on $D$. Nersesyan et al. [18] shows that in one-dimensional spiral model by the interaction of second nearest neighbored which has a simple surface, a new phase is created due to breaking parity symmetry. This calls chiral phase and its order parameter is defined as follows:

$$
\chi^{\alpha}=\left\langle G S\left|\left(\overrightarrow{S_{1}} \times \overrightarrow{S_{2}}\right)^{\alpha}\right| G S\right\rangle,
$$

where $\alpha$ refers to $x-, y$-, $z$-axes. There are two types of gapped and gapless energy spectrum chiral phase [19-21].

For studying the GS phase diagram of the spin chain in the external magnetic field and DM interaction, we calculate the chiral order parameter as follows:

$$
\chi^{y}=\left\langle G S\left|\left(\vec{S}_{1} \times \vec{S}_{2}\right)^{y}\right| G S\right\rangle=\left\langle G S\left|S_{1}^{z} S_{2}^{x}-S_{1}^{x} S_{2}^{z}\right| G S\right\rangle .
$$

In Figure 3, this chiral is plotted as a function of $h$ for different values of $D=0.1,0.5,1.0$. As it can be seen, in the absence of DM interaction, the chiral order parameter does not exist in the ground state of the system. When external magnetic field applies, the order parameter changes

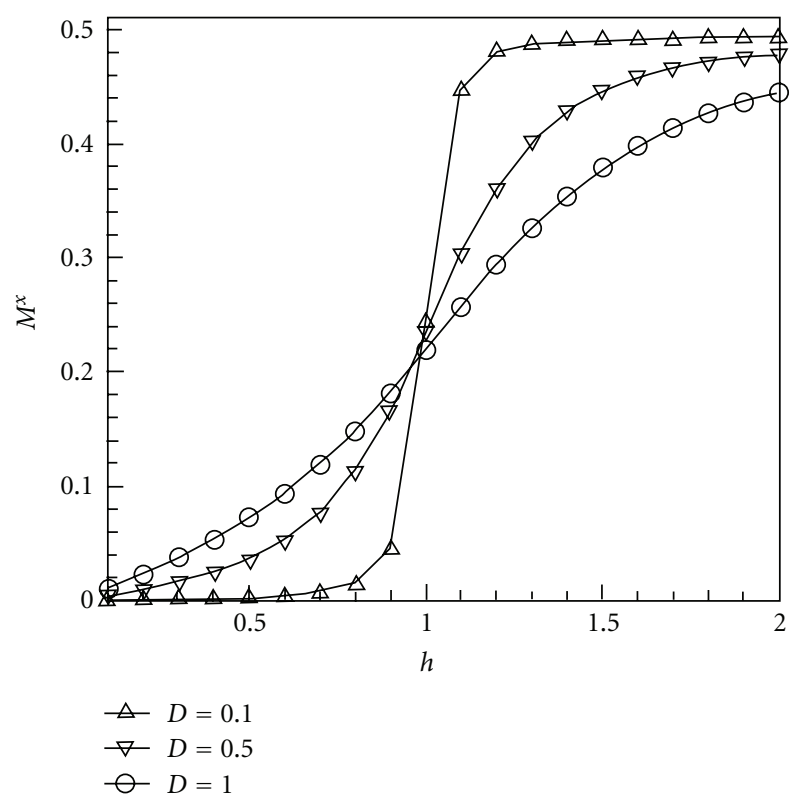

Figure 1: Magnetization along applied field, $M^{x}$, as a function of external field $h$, for exchange constant $J=1$ and different values of DM parameter $D=0.1,0.5,1.0$.

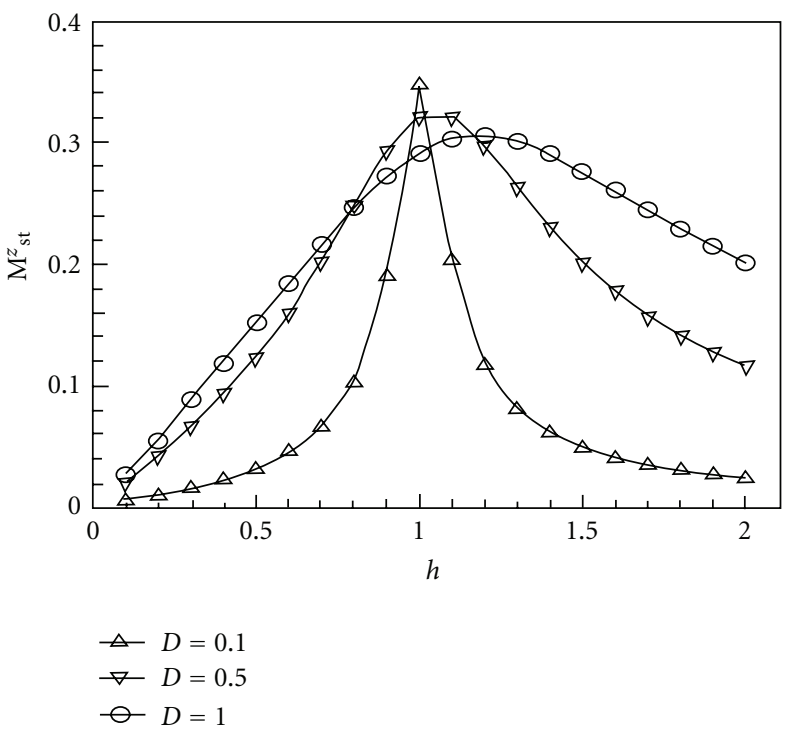

FIGURE 2: Staggered magnetization perpendicular to the plan consists of applied magnetic field and DM vector as a function of external magnetic field for exchange energy $J=1$ and different values of DM interaction $D=0.1,0.5,1.0$.

and starts to increase by increasing the magnetic field. By continuing increase the magnetic field $h$, chiral order parameter will decrease and for sufficiently values of it will be disappeared. This is a state at which the system is in the saturated magnetization phase and the results are in agreement with analytical results of Section 2. 


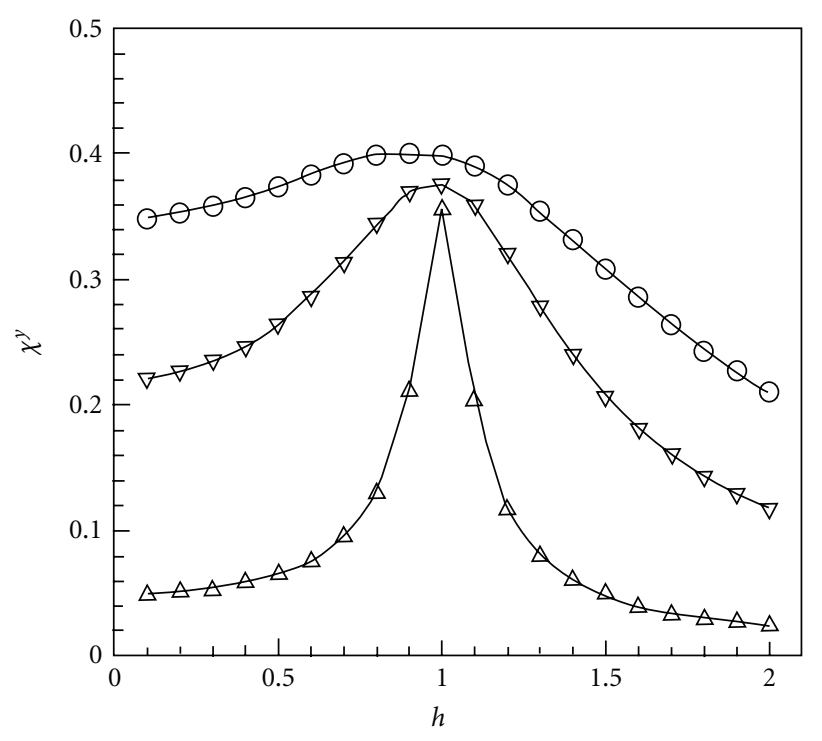

Figure 3: Chiral order parameter in $y$ direction, $\chi^{y}$ as a function of $h$ for $J=1$ and different values of DM interaction parameter $D=0.1,0.5,1.0$.

\section{Three Particle Spin-(1/2) Systems}

Consider a three-particle spin-(1/2) system whose Hamiltonian is as follows:

$$
\begin{aligned}
H= & J\left(\vec{S}_{1} \cdot \vec{S}_{2}+\vec{S}_{2} \cdot \vec{S}_{3}\right) \\
& +D\left(\vec{S}_{1} \times \vec{S}_{2}+\vec{S}_{2} \times \vec{S}_{3}\right) \\
& -h\left(S_{1}^{x}+S_{2}^{x}+S_{3}^{x}\right) .
\end{aligned}
$$

In order to solve the problem, we choose the [base kets] of the Hilbert space as eigenstates of the operator $S_{1}^{z} S_{2}^{z} S_{3}^{z}$ as follows that are

$$
\begin{aligned}
& S_{1}^{z} S_{2}^{z} S_{3}^{z}|\uparrow \uparrow \uparrow\rangle=\frac{1}{8}|\uparrow \uparrow \uparrow\rangle, \\
& S_{1}^{z} S_{2}^{z} S_{3}^{z}|\uparrow \uparrow \downarrow\rangle=-\frac{1}{8}|\uparrow \uparrow \downarrow\rangle, \\
& S_{1}^{z} S_{2}^{z} S_{3}^{z}|\uparrow \downarrow \uparrow\rangle=-\frac{1}{8}|\uparrow \downarrow \uparrow\rangle, \\
& S_{1}^{z} S_{2}^{z} S_{3}^{z}|\downarrow \uparrow \uparrow\rangle=-\frac{1}{8}|\downarrow \uparrow \uparrow\rangle, \\
& S_{1}^{z} S_{2}^{z} S_{3}^{z}|\uparrow \downarrow \downarrow\rangle=\frac{1}{8}|\uparrow \downarrow \downarrow\rangle, \\
& S_{1}^{z} S_{2}^{z} S_{3}^{z}|\downarrow \downarrow \uparrow\rangle=\frac{1}{8}|\downarrow \downarrow \uparrow\rangle, \\
& S_{1}^{z} S_{2}^{z} S_{3}^{z}|\downarrow \uparrow \downarrow\rangle=\frac{1}{8}|\downarrow \uparrow \downarrow\rangle, \\
& S_{1}^{z} S_{2}^{z} S_{3}^{z}|\downarrow \downarrow \downarrow\rangle=-\frac{1}{8}|\downarrow \downarrow \downarrow\rangle .
\end{aligned}
$$

In these states, the Hamiltonian matrix is

$$
H=\left(\begin{array}{cccccccc}
A & B & C & 0 & B & 0 & 0 & 0 \\
B & 0 & A & F & 0 & B & 0 & 0 \\
C & 0 & -A & C & A & 0 & C & 0 \\
0 & F & 0 & 0 & 0 & A & 0 & C \\
B & 0 & A & 0 & -A & B & F & 0 \\
0 & B & 0 & A & B & 0 & A & B \\
0 & 0 & C & 0 & F & 0 & 0 & C \\
0 & 0 & 0 & C & 0 & B & C & A
\end{array}\right) .
$$

In which

$$
A=\frac{J}{4} \longrightarrow B=\frac{D}{4}-\frac{h}{2} \longrightarrow C=-\frac{D}{2}-\frac{h}{2} \longrightarrow F=-\frac{h}{2}
$$

We diagonalzed the Hamiltonian and found and the ground state of the system. Then order parameters as follows:

$$
\begin{aligned}
M_{s t}^{z} & =\frac{1}{2}\left\langle G S\left|S_{1}^{x}+S_{2}^{x}+S_{3}^{x}\right| G S\right\rangle, \\
M_{s t}^{z} & =\frac{1}{2}\left\langle G S\left|S_{1}^{z}-S_{2}^{z}+S_{3}^{z}\right| G S\right\rangle, \\
\chi^{y} & =\left\langle G S\left|\left(\overrightarrow{S_{1}} \times \overrightarrow{S_{2}}\right)^{y}+\left(\overrightarrow{S_{2}} \times \overrightarrow{S_{3}}\right)^{y}\right| G S\right\rangle,
\end{aligned}
$$

are calculated for this system, numerically that are shown in Figures 4 and 5. In the three spin case, we find the same behavior which we do not show. Specially three-particle spin-(1/2) system has exactly the same results of two particle system due to defining the order parameters in the particle number unit. In the XXZ model $h<h_{c}$, the magnetization has some of the quantum fluctuation and the system will be in the magnetic field direction but the magnetization cannot be seen due to the symmetry breakdown in the finite systems.

\section{Conclusion}

In summary, we have studied Heisenberg model with added Dzyaloshinskii-Moriya interaction in the presence of uniform transverse magnetic field. To this end, we have first implemented a uniform rotation about $x$-axis in order to gauge away the added Dzyaloshinskii-Moriya interaction and produce an XXZ model with uniform magnetic and a staggered field normal to $\vec{D}-\vec{h}$ surface. We have chosen blocks of two and three spins to be able to solve the model exactly and have also shown how this scheme has capability to capture the right physics. We plotted magnetization, staggered magnetization, and chirality versus magnetic for different DM interactions strength. In the absence of DM interactions there is a sharp change from zero magnetization at $h<h_{c}$ to saturated region $h>h_{c}$, [but increasing DM interaction favor to produce a nonzero magnetization at $h<h_{c}$ region] and there is no sharp transient any more. Staggered magnetization perpendicular to the plan consists of applied magnetic field and DM vector shows spin-flop ordering. The physical systems 


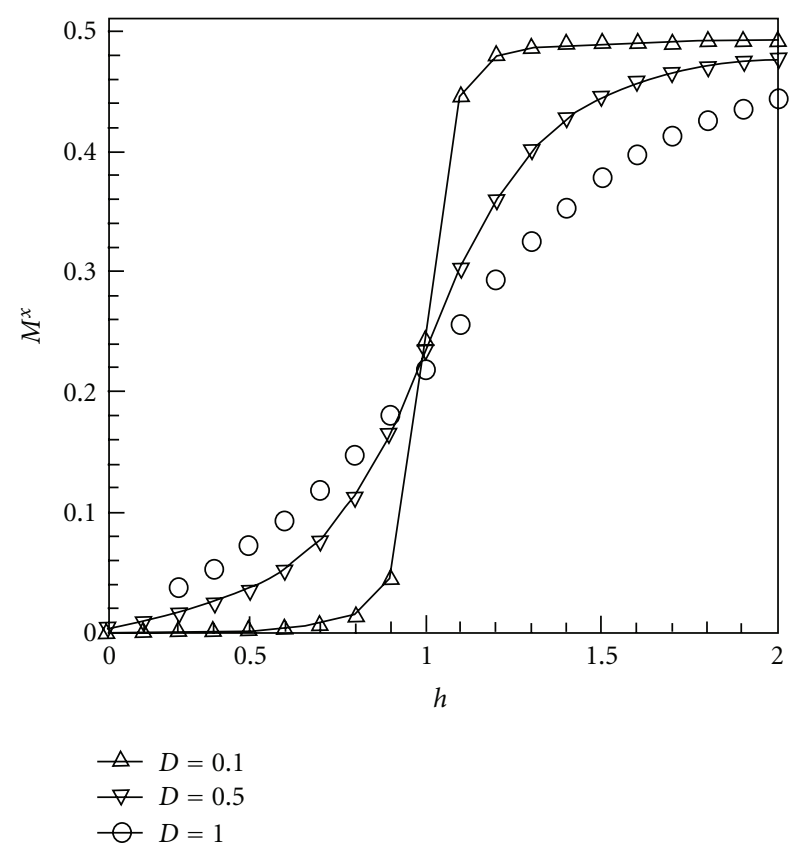

Figure 4: Magnetization along applied field, $M^{x}$ for three particle, as a function of external field $h$, for exchange constant $J=1$ and different values of DM parameter $D=0.1,0.5,1.0$.

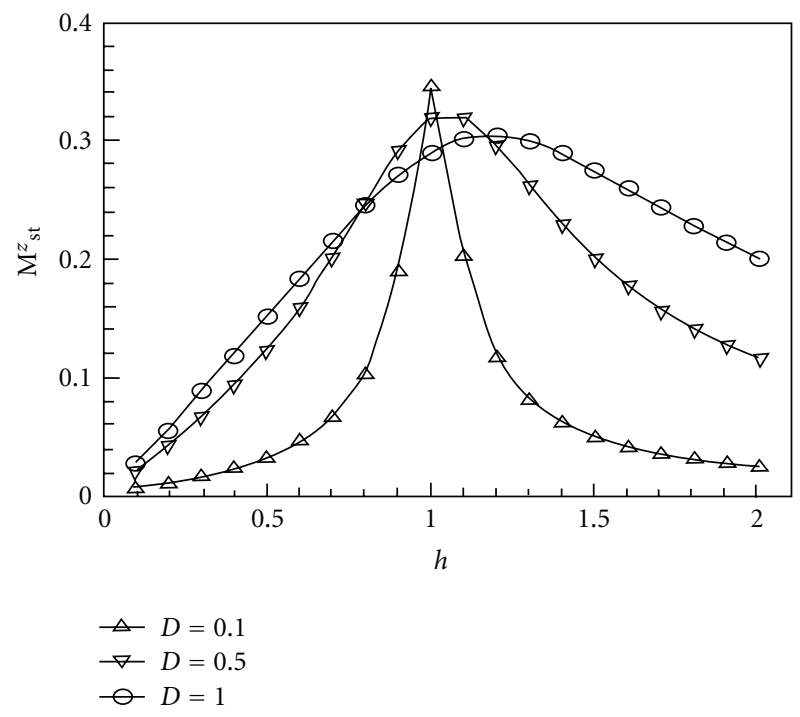

FIGURE 5: Staggered magnetization perpendicular to the plan consists of applied magnetic field and DM vector as a function of external magnetic field for exchange energy $J=1$ and different values of DM interaction $D=0.1,0.5,1.0$.

which prefer spiral chiral configuration usually have competing interactions. For example, when the nearest neighbor (NN) exchange interaction is weak, the next nearest neighbor (NNN) interaction or even Dzyaloshinskii-Moriya (DM) interaction becomes relatively significant. So, we measure chirality in our model versus magnetic field. Our calculations show that in the absence of DM interaction, the chiral order parameter is not in the ground state of the system. But by turning external magnetic field on, the order parameter will be changed and will be increased by increasing the magnetic field. Continuing the increase of magnetic decreases the chiral order and for sufficient values of it will disappear.

\section{References}

[1] C. N. Yang and C. P. Yang, "One-dimensional chain of anisotropic spin-spin interactions. I. Proof of Bethe's hypothesis for ground state in a finite system," Physical Review B, vol. 150, no. 1, pp. 321-327, 1966.

[2] V. L. Pokrovsky and A. L. Talapov, "theory of two-dimensional incommensurate crystals," Soviet Physics, vol. 51, pp. 134-148, 1980.

[3] R. B. Griffiths, "Magnetization curve at zero temperature for the antiferromagnetic heisenberg linear chain," Physical Review, vol. 133, no. 3, pp. A768-A775, 1964.

[4] D. C. Dender, P. R. Hammar, D. H. Reich, C. Broholm, and G. Aeppli, "Direct observation of field-induced incommensurate fluctuations in a one-dimensional S = 1/2 antiferromagnet," Physical Review Letters, vol. 79, no. 9, pp. 1750-1753, 1997.

[5] J. Sirker, A. Weisse, and O. P. Sushkov, "The field-induced magnetic ordering transition in $\mathrm{TlCuCl}_{3}$," Journal of the Physical Society of Japan, vol. 74, pp. 129-134, 2005.

[6] T. Sakai and H. Shiba, "Numerical study of a model for NENP: one-dimensional $\mathrm{S}=1$ antiferromagnet in a staggered field," Journal of the Physical Society of Japan, vol. 63, no. 3, pp. 867871, 1994.

[7] G. Chaboussant, M. H. Julien, Y. Fagot-Revurat et al., "Zero temperature phase transitions in spin-ladders: phase diagram and dynamical studies of $\mathrm{Cu}_{2}\left(\mathrm{C}_{5} \mathrm{H}_{12} \mathrm{~N}_{2}\right)_{2} \mathrm{Cl}_{4}$," European Physical Journal B, vol. 6, no. 2, pp. 167-181, 1998.

[8] H. Kageyama, K. Yoshimura, R. Stern et al., "Exact dimer ground state and quantized magnetization plateaus in the twodimensional spin system $\mathrm{SrCu}_{2}\left(\mathrm{BO}_{3}\right)_{2}$," Physical Review Letters, vol. 82, no. 15, pp. 3168-3171, 1999.

[9] O. Cépas, K. Kakurai, L. P. Regnault et al., "Dzyaloshinskimoriya interaction in the $2 \mathrm{D}$ spin gap system $\mathrm{SrCu}_{2}\left(\mathrm{BO}_{3}\right)_{2}$," Physical Review Letters, vol. 87, no. 16, Article ID 167205, pp. $1-4,2001$.

[10] M. Jaime, V. F. Correa, N. Harrison et al., "Magnetic-fieldinduced condensation of triplons in Han Purple pigment $\mathrm{BaCuSi}_{2} \mathrm{O}_{6}$," Physical Review Letters, vol. 93, no. 8, Article ID 087203, 2004.

[11] I. Dzyaloshinsky, "A thermodynamic theory of "weak" ferromagnetism of antiferromagnetics," Journal of Physics and Chemistry of Solids, vol. 4, no. 4, pp. 241-255, 1958.

[12] T. Moriya, "New mechanism of anisotropic superexchange interaction," Physical Review Letters, vol. 4, no. 5, pp. 228-230, 1960.

[13] M. Oshikawa and I. Affleck, "Field-induced gap in S = 1/2 antiferromagnetic chains," Physical Review Letters, vol. 79, no. 15, pp. 2883-2886, 1997.

[14] M. Sato and M. Oshikawa, "Coupled S=1/2 Heisenberg antiferromagnetic chains in an effective staggered field," Physical Review B, vol. 69, no. 5, Article ID 54406, 12 pages, 2004.

[15] Y. Onose, T. Ideue, H. Katsura, Y. Shiomi, N. Nagaosa, and Y. Tokura, "Observation of the magnon hall effect," Science, vol. 329, no. 5989, pp. 297-299, 2010. 
[16] O. A. Tretiakov and A. Abanov, "Current driven mnagnetization dynamics in ferromagnetic nanowires with a dzyaloshinskii-moriya interaction," Physical Review Letters, vol. 105, no. 15, Article ID 157201, 2010.

[17] I. G. Bostrem, J. I. Kishine, and A. S. Ovchinnikov, "Theory of spin current in chiral helimagnets," Physical Review B, vol. 78, no. 6, Article ID 064425, 2008.

[18] A. A. Nersesyan, A. O. Gogolin, and F. H. L. Eler, "Incommensurate spin correlations in spin-1/2 frustrated two-Leg heisenberg ladders," Physical Review Letters, vol. 81, no. 4, pp. 910-913, 1998.

[19] M. Kaburagi, H. Kawamura, and T. Hikihara, "Spin and chiral orderings of frustrated quantum spin chains," Journal of the Physical Society of Japan, vol. 68, no. 10, pp. 3185-3188, 1999.

[20] T. Hikihara, M. Kaburagi, and H. Kawamura, "Ground-state phase diagrams of frustrated spin-S XXZ chains: chiral ordered phases," Physical Review B, vol. 63, no. 17, pp. 174430117443011, 2001.

[21] M. Takahashi, Thermodynamics of One Dimensional Solvable Models, Cambridge University Press, 1999. 

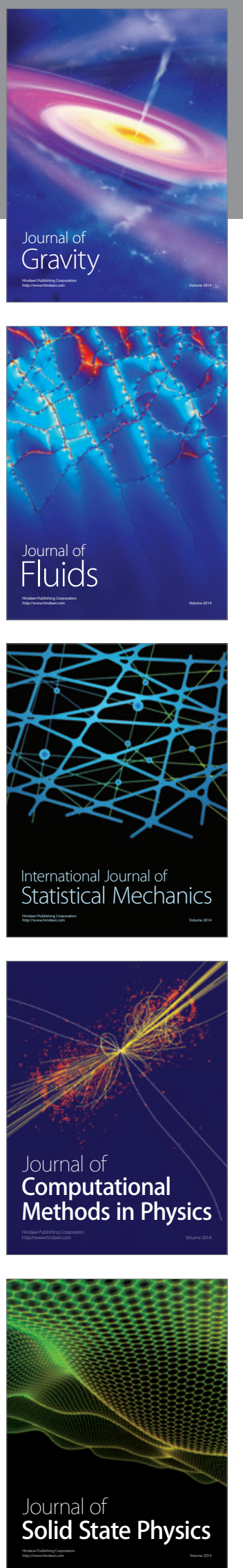
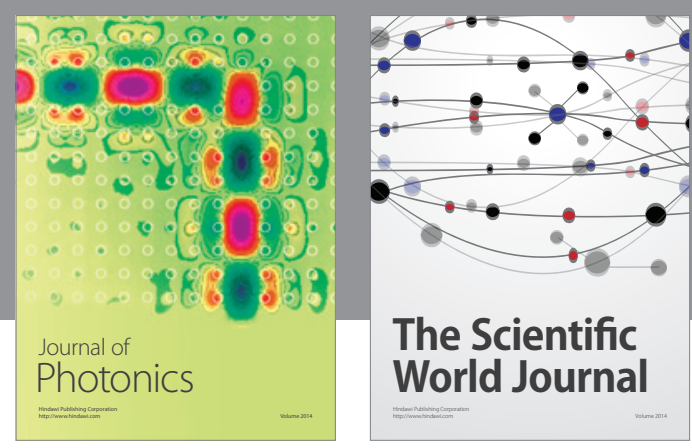

The Scientific World Journal

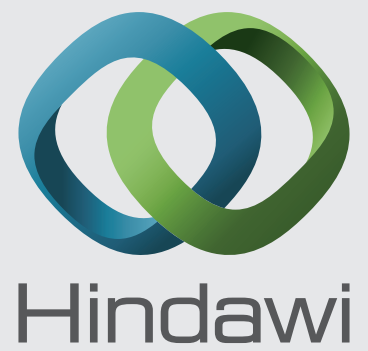

Submit your manuscripts at http://www.hindawi.com
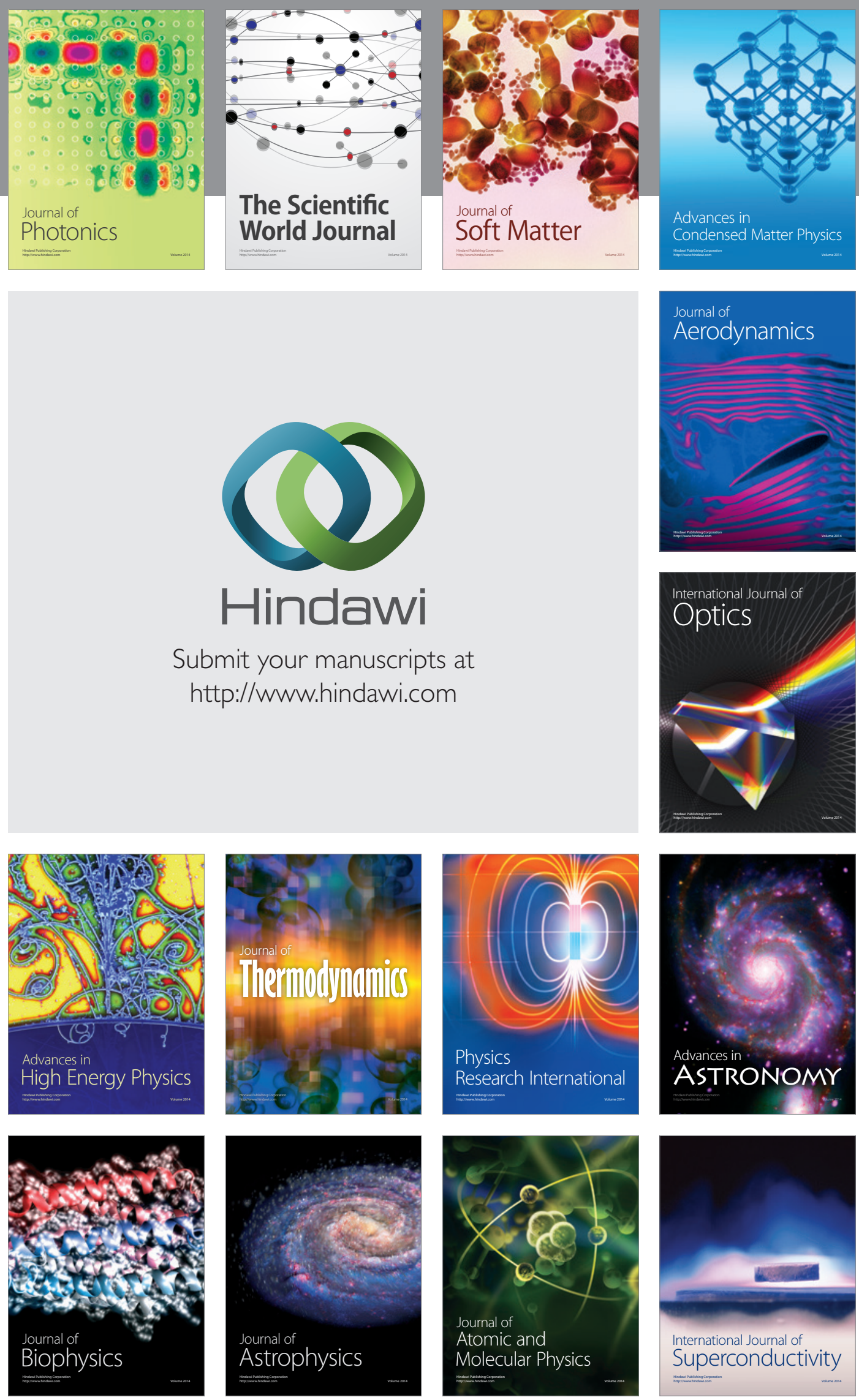
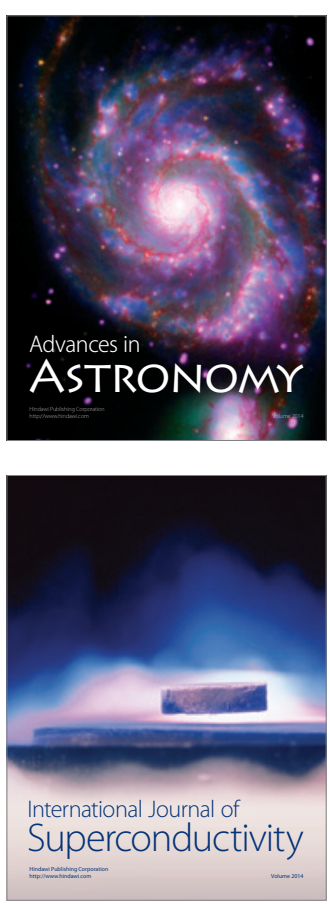